\title{
Some results on quadratic stability of switched systems with interval uncertainty
}

Ezra Zeheb*, Oliver Mason†, Selim Solmaz ${ }^{\ddagger}$ and Robert Shorten ${ }^{\S}$

\begin{abstract}
In this paper we present some results on the quadratic stability of switched systems with uncertain parameters. We show that the quadratic stability of a class of switched uncertain systems may be readily verified using simple algebraic conditions.
\end{abstract}

Keywords: Stability theory; Switched linear systems; Interval uncertainty; Lyapunov functions.

\section{Introduction}

In this paper we consider the stability of switched linear systems of the form $\Sigma: \dot{x}(t)=A(t) x(t)\left(\right.$ where $\left.x(t) \in \mathbb{R}^{n}, A(t) \in \mathbb{R}^{n \times n}, A(t) \in\left\{A_{1}, \ldots, A_{m}\right\}\right)$.

${ }^{*}$ E. Zeheb is with the Technion-Israel Institute of Technology,Haifa, and Jerusalem College of Engineering, Jerusalem, Israel zeheb@ee.technion.ac.il (joint first author).

${ }^{\dagger}$ O. Mason is with the Hamilton Institute, NUI Maynooth, Co. Kildare, Ireland oliver.mason@nuim.ie (joint first author)

${ }_{\ddagger}^{\ddagger}$ S. Solmaz is with the Hamilton Institute, NUI Maynooth, Co. Kildare, Ireland selim.solmaz@nuim.ie

${ }^{\S} \mathrm{R}$. Shorten is with the Hamilton Institute, NUI Maynooth, Co. Kildare, Ireland robert.shorten@nuim.ie 
One way of establishing the stability of such systems is to show that for some positive definite matrix $P$ the quadratic Lyapunov function $x(t)^{T} P x(t)$ is decreasing in time; namely that $A_{i}^{T} P+P A_{i}<0$ for all $1 \leq i \leq m$. When such a function exists, then the associated LTI systems

$$
\Sigma_{A_{i}}: \dot{x}(t)=A_{i} x(t) \quad 1 \leq i \leq m
$$

are said to have a common quadratic Lyapunov function (CQLF). Recently, motivated by the stability of switched systems Liberzon and Morse (1999), the problem of determining compact conditions for the existence of a CQLF for a finite number of LTI systems has assumed a position of great theoretical importance in the mathematics and engineering communities; see Ando (2001); Shorten and Narendra (2003); Shorten et al. (2003); Cohen and Lewkowicz (2003); Narendra and Balakrishnan (1994); Agrachev and Liberzon (2001) for some of the recent work in this area. Notwithstanding the progress that has been made on the general CQLF existence problem, the problem of determining whether or not a set of LTI systems subject to interval uncertainty has a CQLF has received relatively little attention, despite its obvious considerable practical importance. Our objective in this paper is to study this problem for a restricted class of switching systems subject to interval uncertainty; namely the class of switching systems given by $\dot{x}=A(t) x, A(t) \in\left\{A, A-g h^{T}\right\}$, where $g, h \in \mathbb{R}^{n}$, and the system matrices $A, A-g h^{T}$ are subject to interval uncertainty of the form $\underline{a}_{i j} \leq a_{i j} \leq \bar{a}_{i j}$.

The class of switched linear systems that we study is thus restricted in two ways:

1. We consider switching between two LTI systems, $\Sigma_{A_{1}}, \Sigma_{A_{2}}$;

2. the system matrices $A_{1}$ and $A_{2}$ differ by rank one $\left(A_{2}=A_{1}-g h^{T}\right)$.

The first restriction, although a special case of the general problem of switching between an arbitrary number of LTI systems, is important, has numerous applications, and has been extensively treated in the literature (see e.g. Ooba and Funahashi (1997); Cohen and Lewkowicz (2003)). It is relevant to control systems which include a relay with two states e.g. "on" and "off", or other linear dynamical systems containing a single switch whose position is assumed to take on values from a discrete set of the form $\{0,1\}$ according 
to a certain rule. Also, many saturation systems, hysteresis systems and backlash systems can be described as switching between two linear systems.

Obviously, a necessary condition for the existence of a CQLF for a finite set of LTI systems is that every pair of systems belonging to the set has a CQLF. Moreover, there can exist system classes for which the existence of a CQLF for any pair of systems in a finite family implies the existence of a CQLF for the entire family. This was shown to be the case for the class of second order positive systems in Gurvits et al. (2004). This fact provides further motivation for the study of the problem of CQLF existence for pairs of systems.

With regard to the second restriction, pairs of systems differing by rank one have historically occupied a position of great importance in systems theory, and several classical results on absolute stability for single-input single-output systems such as the Popov and Circle Criteria can be cast naturally in this framework. Also, this class of systems includes pairs of systems whose system matrices are in companion form as a subclass. Furthermore, switching between systems differing by rank one arises in a number of practical applications. For example, in Vilaplana et al. (2005) a control system for four-wheel steering vehicles is described which involves switching between a pair of LTI systems differing by rank one whose parameters are subject to interval uncertainty. It should also be noted that this class of systems has received a considerable amount of attention in the literature Shorten and Narendra (2003); Narendra and Goldwyn (1964); King and Nathanson (2004).

It should be emphasized that, in compensation to these restrictions, this paper extends results for the class of systems under study in a very important direction. Every mathematical model of a physical system is inaccurate and includes uncertainties. These are either inherent to the model or a result of measurement inaccuracies or environmental changes, etc. These uncertainties can often be characterized by interval parameters in the model. Such "interval models" are, however, difficult to analyze and thus are frequently neglected unjustifiably. Alternatively, numerical methods are used. In this paper we treat such interval uncertainty in a systematic analytic way which is independent of the uncertainty. 


\section{Preliminary results}

Throughout this note $\mathbb{R}$ and $\mathbb{C}$ denote the fields of real and complex numbers respectively. We denote $n$-dimensional real Euclidean space by $\mathbb{R}^{n}$ and the space of $n \times n$ matrices with real entries by $\mathbb{R}^{n \times n}$. For a vector $x$ in $\mathbb{R}^{n}, x_{i}$ denotes the $i^{\text {th }}$ component of $x$ and for $A$ in $\mathbb{R}^{n \times n}$, we denote the entry in the $(i, j)$ position by $a_{i j}$.

\section{CQLF existence for systems differing by rank one:}

The classical single-input single-output (SISO) Circle Criterion Narendra and Goldwyn (1964) gives a necessary and sufficient condition for two fixed coefficient LTI systems with system matrices in companion form to have a CQLF. Formally, if $A, A-g h^{T}$ are two Hurwitz matrices in $\mathbb{R}^{n \times n}$ in companion form, then the LTI systems $\Sigma_{A}, \Sigma_{A-g h^{T}}$ have a CQLF if and only if the rational function

$$
1+h^{T}(s I-A)^{-1} g
$$

is strictly positive real (SPR), meaning that

$$
1+\operatorname{Re}\left\{h^{T}(j \omega I-A)^{-1} g\right\}>0 \text { for all } \omega \in \mathbb{R} .
$$

Moreover, Meyer's extension of the KYP Lemma in Meyer (1966) established that (2) is also sufficient for CQLF existence for two LTI systems $\Sigma_{A}$, $\Sigma_{A-g h^{T}}$ where $A, A-g h^{T}$ are Hurwitz matrices differing by rank one, but not necessarily in companion form. Condition (2) is also necessary for CQLF existence in this case. This can be seen by combining the known fact that if the LTI stable systems $\Sigma_{A_{1}}, \Sigma_{A_{2}}$ have a CQLF, then the product $A_{1} A_{2}$ has no real negative eigenvalues with the result in Shorten et al. (2004b) that if $A, A-g h^{T}$ are Hurwitz matrices, then (2) is equivalent to the product $A\left(A-g h^{T}\right)$ having no real negative eigenvalues.

Theorem 2.1 Let $A, A-g h^{T}$ be Hurwitz matrices in $\mathbb{R}^{n \times n}$, where $g, h \in \mathbb{R}^{n}$. Then

$$
1+\operatorname{Re}\left\{h^{T}(j \omega I-A)^{-1} g\right\}>0 \text { for all } \omega \in \mathbb{R}
$$

if and only if the matrix product $A\left(A-g h^{T}\right)$ has no negative real eigenvalues. 
In the paper Shorten et al. (2004a), a number of implications of this equivalence for multiplier-type stability results were described, and one immediate consequence of it is the following time-domain condition for CQLF existence.

Theorem 2.2 Shorten et al. (2004b) Let A, A-ght be two Hurwitz matrices in $\mathbb{R}^{n \times n}$ where $g, h$ are vectors in $\mathbb{R}^{n}$. A necessary and sufficient condition for the existence of a common quadratic Lyapunov function for the LTI systems, $\Sigma_{A}$, and $\Sigma_{A-g h^{T}}$ is that the matrix product $A\left(A-g h^{T}\right)$ does not have any negative real eigenvalues.

In the remainder of this paper, we shall show how Theorem 2.2 may be used to obtain results on CQLF existence for pairs of LTI systems subject to interval uncertainty.

\section{Kharitonov's theorem and rational transfer functions:}

In the next section, we shall make use of a version of Kharitonov's Theorem for rational functions that was derived in Chapellat et al. (1991). Let $\mathcal{P}$ be the family of interval polynomials of order $n$ given by

$$
p(s)=p_{0}+p_{1} s+\cdots+p_{n} s^{n},
$$

where $\underline{p}_{i} \leq p_{i} \leq \bar{p}_{i}$ for $1 \leq i \leq n$. Then define the four Kharitonov polynomials associated with $\mathcal{P}$ :

$$
\begin{aligned}
& k_{1}^{\mathcal{P}}(s)=\underline{p}_{0}+\underline{p}_{1} s+\bar{p}_{2} s^{2}+\bar{p}_{3} s^{3}+\cdots \\
& k_{2}^{\mathcal{P}}(s)=\underline{p}_{0}+\bar{p}_{1} s+\bar{p}_{2} s^{2}+\underline{p}_{3} s^{3}+\underline{p}_{4} s^{4} \cdots \\
& k_{3}^{\mathcal{P}}(s)=\bar{p}_{0}+\underline{p}_{1} s+\underline{p}_{2} s^{2}+\bar{p}_{3} s^{3}+\bar{p}_{4} s^{4}+\cdots \\
& k_{4}^{\mathcal{P}}(s)=\bar{p}_{0}+\bar{p}_{1} s+\underline{p}_{2} s^{2}+\underline{p}_{3} s^{3}+\cdots
\end{aligned}
$$

If $\mathcal{P}$ and $\mathcal{Q}$ are two families of interval polynomials of order $n$ and $m$ respectively with $n \leq m$, then $\mathcal{P} / \mathcal{Q}$ denotes the family of proper rational functions of the form

$$
\frac{p(s)}{q(s)}
$$

where $p \in \mathcal{P}$ and $q \in \mathcal{Q}$. The following result on the strict positive realness of all of the rational functions in $\mathcal{P} / \mathcal{Q}$ was derived in Chapellat et al. (1991). 
Theorem 2.3 Every transfer function in the family $\mathcal{P} / \mathcal{Q}$ is strictly positive real if and only if the following eight transfer functions are strictly positive real.

$$
\begin{aligned}
& \frac{k_{2}^{\mathcal{P}}(s)}{k_{1}^{\mathcal{Q}}(s)}, \frac{k_{3}^{\mathcal{P}}(s)}{k_{1}^{\mathcal{Q}}(s)}, \frac{k_{1}^{\mathcal{P}}(s)}{k_{2}^{\mathcal{Q}}(s)}, \frac{k_{4}^{\mathcal{P}}(s)}{k_{2}^{\mathcal{Q}}(s)}, \\
& \frac{k_{1}^{\mathcal{P}}(s)}{k_{3}^{\mathcal{Q}}(s)}, \frac{k_{4}^{\mathcal{P}}(s)}{k_{3}^{\mathcal{Q}}(s)}, \frac{k_{2}^{\mathcal{P}}(s)}{k_{4}^{\mathcal{Q}}(s)}, \frac{k_{3}^{\mathcal{P}}(s)}{k_{4}^{\mathcal{Q}}(s)},
\end{aligned}
$$

where $k_{i}^{\mathcal{P}}, k_{i}^{\mathcal{Q}}, 1 \leq i \leq 4$, are the Kharitonov polynomials corresponding to the interval polynomial families $\mathcal{P}$ and $\mathcal{Q}$ respectively.

\section{Positivity of multi-variable polynomials:}

Finally for this section, we present the following theorem from Walach and Zeheb (1980) on the positivity of multi-variable polynomials. Later, we shall combine this result with Theorem 2.2 to obtain necessary and sufficient conditions for CQLF existence for certain classes of systems subject to interval uncertainty. Theorem 2.4 below provides necessary and sufficient conditions for a polynomial $p(x)$ of $n$-variables (defined for $x \in \mathbb{R}^{n}$ ) to be positive for all $x$ in a region $D^{n}$ of the form

$$
\left\{x \in \mathbb{R}^{n}: \underline{x}_{i} \leq x_{i} \leq \bar{x}_{i}\right\},
$$

where the end-points $\underline{x}_{i}, \bar{x}_{i}$, may be finite or infinite.

Theorem 2.4 Walach and Zeheb (1980) Let $p(x)$ be a real-coefficient polynomial of $n$-variables, and $D^{n}$ be a region in $\mathbb{R}^{n}$ of the form (10). Then $p(x)>0$ for all $x \in D^{n}$ if and only if:

(i) the polynomials obtained by setting the value of one of the variables $x_{i}$ to one of the end-points $\underline{x}_{i}$ or $\bar{x}_{i}$ are all positive for the permitted values of the remaining variables $x_{1}, \ldots, x_{i-1}, x_{i+1}, \ldots, x_{n}$; This should hold for all indices $i$.

(ii) there is no $x \in D^{n}$ satisfying the $n$ equations

$$
\begin{aligned}
p(x) & =0 \\
\frac{\partial p}{\partial x_{i}}(x) & =0 \quad \text { for } 1 \leq i \leq n-1 .
\end{aligned}
$$




\section{Main results}

\subsection{CQLF existence for general pairs of interval ma- trices differing by rank one}

Let $\mathcal{A}$ be a real interval matrix family in $\mathbb{R}^{n \times n}$ of the form

$$
\mathcal{A}=\left\{A \in \mathbb{R}^{n \times n}: \underline{a}_{i j} \leq a_{i j} \leq \bar{a}_{i j} \text { for } 1 \leq i, j \leq n\right\},
$$

and let $g, h$ be two fixed vectors in $\mathbb{R}^{n}$. Theorem 3.1 below is concerned with the following question.

Determine necessary and sufficient conditions for any pair of LTI systems $\Sigma_{A}, \Sigma_{A-g h^{T}}$ where $A \in \mathcal{A}$ to have a CQLF.

It is important to note that in this situation, the interval uncertainty is in the matrix $A$, while the difference $g h^{T}$ is fixed. In the next subsection, we consider two systems in companion form, each of which is independently subject to interval uncertainty.

We shall assume that all of the matrices $A, A-g h^{T}$ for $A \in \mathcal{A}$ are Hurwitz. This assumption can be validated by known analytic methods such as in Sezer and Siljak (1994); Rojas and Collada (1994); Zeheb (1990). Admittedly, the computational complexity of the check is high for large $n$. Also, in the statement of the next theorem, the notation $\mathcal{A}_{1}$ is used to denote the set of all matrices belonging to $\mathcal{A}$ where one entry $a_{i j}$ is set equal to one of the interval endpoints $\underline{a}_{i j}$ or $\bar{a}_{i j}$.

Theorem 3.1 Let $\mathcal{A}$ be a real interval matrix family of the form (12) and let $g, h$ be two fixed vectors in $\mathbb{R}^{n}$ such that all matrices $A, A-g h^{T}$ for $A \in \mathcal{A}$ are Hurwitz. Let $f\left(s, a_{i j}\right)$ be the polynomial in $n^{2}+1$ variables given by

$$
f\left(s, a_{i j}\right)=\operatorname{det}\left(s I-A\left(A-g h^{T}\right)\right) .
$$

Then the systems $\Sigma_{A}, \Sigma_{A-g h^{T}}$ have a CQLF for each $A$ in $\mathcal{A}$ if and only if:

(i) there is no solution to the set of simultaneous equations

$$
\begin{aligned}
f\left(s, a_{i j}\right) & =0 \\
\frac{\partial f}{\partial a_{i j}}\left(s, a_{i j}\right) & =0
\end{aligned}
$$


in the range $-\infty<s<0, \underline{a}_{i j} \leq a_{i j} \leq \bar{a}_{i j} ;$

(ii) for each $A$ in the reduced family $\mathcal{A}_{1}$, there is a CQLF for the systems $\Sigma_{A}, \Sigma_{A-g h^{T}}$.

Proof: First of all, Theorem 2.2 establishes that for fixed Hurwitz matrices $A, A-g h^{T}$, the systems $\Sigma_{A}, \Sigma_{A-g h^{T}}$ have a CQLF if and only if the matrix product $A\left(A-g h^{T}\right)$ has no negative real eigenvalues. Thus, $\Sigma_{A}, \Sigma_{A-g h^{T}}$ will have a CQLF for any $A$ in $\mathcal{A}$ if and only if $A\left(A-g h^{T}\right)$ has no negative real eigenvalues for all $A$ in $\mathcal{A}$. Equivalently, the polynomial $f\left(s, a_{i j}\right)$ must have the same sign for all $s<0$ and all $a_{i j}$ with $\underline{a}_{i j} \leq a_{i j} \leq \bar{a}_{i j}$. In fact, for $n$ even (odd) we must have $f\left(s, a_{i j}\right)>0\left(f\left(s, a_{i j}\right)<0\right)$ for all $s<0$, and $\underline{a}_{i j} \leq a_{i j} \leq \bar{a}_{i j}$. We shall prove the result for the case when $n$ is even. The proof for odd $n$ proceeds identically.

It follows from Theorem 2.4 that $f\left(s, a_{i j}\right)>0$ for all $s<0$, and $\underline{a}_{i j} \leq a_{i j} \leq \bar{a}_{i j}$ if and only if:

(i) there is no solution to the set of equations (13) within the range $-\infty<$ $s<0, \underline{a}_{i j} \leq a_{i j} \leq \bar{a}_{i j} ;$

(ii) all of the polynomials obtained by fixing the value of one of the variables $a_{k l}$ to one of the endpoints $\underline{a}_{k l}$ or $\bar{a}_{k l}$ are positive within the allowed ranges for the remaining variables.

Condition (i) above is obviously equivalent to condition (i) in the statement of the theorem while condition (ii) is equivalent to requiring that for all matrices $A$ belonging to the family $\mathcal{A}_{1}$, the product $A\left(A-g h^{T}\right)$ has no negative real eigenvalues, or equivalently by Theorem 2.2 that the systems $\Sigma_{A}, \Sigma_{A-g h^{T}}$ have a CQLF. This completes the proof.

\section{Remarks:}

(i) Condition (ii) of Theorem 3.1 replaces the original problem with a finite number of CQLF existence problems for interval matrix families. However, the number of uncertain parameters in each of these problems has been reduced by one, as one of the entries $a_{i j}$ is fixed in each case. Repeatedly applying the theorem to these reduced problems will eventually lead to a finite number of fixed coefficient CQLF existence problems, each of which can be solved using Theorem 2.2. 
(ii) Condition (i) involves $n^{2}+1$ polynomial equations in the $n^{2}+1$ variables $s, a_{i j}, 1 \leq i, j \leq n$. There exist analytic systematic methods to reduce this task, by a finite number of steps, to the problem of solving polynomial equations with fixed coefficients. One such method uses Grobner Bases Buchberger (1985). Another method is based on resultants and Sylvester matrices Bocher (1964). While from the computational viewpoint this condition is difficult to check in general, for some classes of system it may be possible to verify that it is automatically satisfied, thus reducing the problem of CQLF existence under interval uncertainty to a number of fixed coefficient CQLF existence problems. We shall next give an example where this is indeed the case.

(iii) The significance of this result is that the problem, which is on one hand very difficult and on the other hand very important, of uncertain matrices is now dealt by a clear and fixed algorithm independent of uncertainty. This result has been achieved by using a combination of two results from two different areas and applications.

Corollary 3.1 Let $\mathcal{A}$ be an interval matrix family in companion form in $\mathbb{R}^{2 \times 2}$ consisting of matrices of the form

$$
A=\left(\begin{array}{cc}
0 & 1 \\
-a_{1} & -a_{2}
\end{array}\right)
$$

where $\underline{a}_{i} \leq a_{i} \leq \bar{a}_{i}$ for $i=1,2$, and let $g=(0,1)^{T}$ and $h=\left(h_{1}, h_{2}\right)^{T}$ be fixed. Moreover, assume that for every $A \in \mathcal{A}, A$ and $A-g h^{T}$ are both Hurwitz. Then, for every $A \in \mathcal{A}$, the LTI systems $\Sigma_{A}, \Sigma_{A-g h^{T}}$ have a $C Q L F$ if and only if $\Sigma_{A_{i}}, \Sigma_{A_{i}-g h^{T}}$ have a CQLF for $1 \leq i \leq 4$, where $A_{1}, \ldots, A_{4}$ are the four fixed coefficient matrices obtained by setting $a_{i}$ equal to $\underline{a}_{i}$ or $\bar{a}_{i}$ for $i=1,2$.

Proof: From Theorem 3.1, it is enough to show that there can be no solution to the equations (13) in the range $\underline{a}_{i} \leq a_{i} \leq \bar{a}_{i},-\infty<s<0$. By assumption, all matrices $A, A-g h^{T}$ for $A \in \mathcal{A}$ are Hurwitz. It follows from this that for each $A$ belonging to $\mathcal{A}, a_{i}>0$ and $a_{i}+h_{i}>0$ for $i=1,2$. In other words, $\underline{a}_{i}>\max \left\{0,-h_{i}\right\}$ for $i=1,2$.

Now the polynomial $f\left(s, a_{i j}\right)=\operatorname{det}\left(s I-A\left(A-g h^{T}\right)\right)$ is given by

$$
f\left(s, a_{i j}\right)=s^{2}+s\left(2 a_{1}+h_{1}-a_{2}\left(a_{2}+h_{2}\right)\right)+a_{1}\left(a_{1}+h_{1}\right) .
$$


Thus, in the current situation, condition (i) requires that

$$
\begin{aligned}
f\left(s, a_{1}, a_{2}\right) & =0 \\
\frac{\partial f}{\partial a_{1}}=2 s+2 a_{1}+h_{1} & =0 \\
\frac{\partial f}{\partial a_{2}}=-s\left(2 a_{2}+h_{2}\right) & =0 .
\end{aligned}
$$

But as $a_{2}>0$ and $a_{2}+h_{2}>0$, the only possible solution to the third equation in (15) under the hypotheses of the corollary is $s=0$. The result now follows immediately.

\subsection{CQLF existence for interval matrices in companion form}

In this subsection, we derive a result on CQLF existence for a pair of interval matrix families in companion form. At first, this may appear to be a special case of the problem considered in the previous subsection. However, previously we assumed a fixed difference $g h^{T}$ between the matrices whereas here we are considering the CQLF problem for two families of companion matrices each of which is independently subject to interval uncertainty. Moreover, the result of this subsection is derived using different considerations and yields necessary and sufficient conditions expressed explicitly in terms of eight fixed coefficient matrices. The result is obtained by combining results from two different areas and applications. For notational convenience, we shall denote the companion matrix

$$
A=\left(\begin{array}{llllr}
0 & 1 & 0 & \ldots & 0 \\
0 & 0 & 1 & \ldots & 0 \\
\vdots & & & & \\
0 & 0 & 0 & \ldots & 1 \\
-a_{0} & -a_{1} & -a_{2} & \ldots & -a_{n-1}
\end{array}\right)
$$

by $C\left(a_{0}, \ldots, a_{n-1}\right)$. Now, consider the matrix families $\mathcal{A}, \mathcal{B}$ consisting of companion matrices $C\left(a_{0}, \ldots, a_{n-1}\right)$ and $C\left(b_{0}, \ldots, b_{n-1}\right)$ respectively, where $\underline{a}_{i} \leq a_{i} \leq \bar{a}_{i}, \underline{b}_{i} \leq b_{i} \leq \bar{b}_{i}$ for $0 \leq i \leq n-1$. In Theorem 3.2 below, we consider the following problem. 
Determine necessary and sufficient conditions for any pair of LTI systems $\Sigma_{A}, \Sigma_{B}$ with $A \in \mathcal{A}, B \in \mathcal{B}$ to have a CQLF.

We are concerned with CQLF existence for pairs of systems $\Sigma_{A}, \Sigma_{B}$ with $A \in \mathcal{A}, B \in \mathcal{B}$. Hence, we shall assume that all of the matrices belonging to the families $\mathcal{A}, \mathcal{B}$ are Hurwitz. The problem of determining whether or not a family of interval matrices consists entirely of Hurwitz matrices has itself been the subject of a considerable amount of research Sezer and Siljak (1994); Rojas and Collada (1994); Zeheb (1990), and in the case of interval matrices in companion form, Kharitonov's Theorem can be used to test for stability.

For the interval matrix family $\mathcal{A}$, construct the four matrices

$$
\begin{aligned}
& A_{1}=C\left(\underline{a}_{0}, \underline{a}_{1}, \bar{a}_{2}, \bar{a}_{3}, \ldots\right) \\
& A_{2}=C\left(\underline{a}_{0}, \bar{a}_{1}, \bar{a}_{2}, \underline{a}_{3}, \underline{a}_{4}, \ldots\right) \\
& A_{3}=C\left(\bar{a}_{0}, \underline{a}_{1}, \underline{a}_{2}, \bar{a}_{3}, \bar{a}_{4}, \ldots\right) \\
& A_{4}=C\left(\bar{a}_{0}, \bar{a}_{1}, \underline{a}_{2}, \underline{a}_{3}, \ldots\right),
\end{aligned}
$$

in analogy with the Kharitonov polynomials given by (4)-(7). The matrices $B_{1}, B_{2}, B_{3}, B_{4}$ are defined in the same manner for the family $\mathcal{B}$. We are now ready to state the main result of this subsection.

Theorem 3.2 Consider the interval matrix families $\mathcal{A}, \mathcal{B}$ given by (16), and assume that all the matrices belonging to $\mathcal{A}, \mathcal{B}$ are Hurwitz. Then for every pair of LTI systems of the form $\Sigma_{A}, \Sigma_{B}$ with $A \in \mathcal{A}, B \in \mathcal{B}$ to have a CQLF, it is necessary and sufficient that none of the eight matrix products

$$
\begin{aligned}
& A_{1} B_{2}, A_{1} B_{3}, A_{2} B_{1}, A_{2} B_{4}, \\
& A_{3} B_{1}, A_{3} B_{4}, A_{4} B_{2}, A_{4} B_{3},
\end{aligned}
$$

has a negative real eigenvalue.

Proof: Let $A=C\left(a_{0}, \ldots, a_{n-1}\right), B=C\left(b_{0}, \ldots, b_{n-1}\right)$ be two matrices in the families $\mathcal{A}$ and $\mathcal{B}$ respectively, and write $B=A-g h^{T}$ where $g=(0,0, \ldots, 1)^{T}$, and $h=\left(b_{0}-a_{0}, \ldots, b_{n-1}-a_{n-1}\right)^{T}$. Then it follows from the Circle Criterion that the LTI systems $\Sigma_{A}, \Sigma_{B}$ have a CQLF if and only if the rational function

$$
1+h^{T}(s I-A)^{-1} g
$$


is strictly positive real.

It can be verified by direct computation that

$$
1+h^{T}(s I-A)^{-1} g=b(s) / a(s)
$$

where the polynomials $a(s), b(s)$ are given by

$$
\begin{aligned}
& a(s)=a_{0}+a_{1} s+\cdots a_{n-1} s^{n-1}+s^{n} \\
& b(s)=b_{0}+b_{1} s+\cdots b_{n-1} s^{n-1}+s^{n} .
\end{aligned}
$$

It now follows that every pair of LTI systems $\Sigma_{A}, \Sigma_{B}$ with $A \in \mathcal{A}, B \in \mathcal{B}$ will have a CQLF if and only if all of the rational functions $b(s) / a(s)$ are strictly positive real where $a(s)$ and $b(s)$ belong to the interval polynomial families

$$
\begin{aligned}
& a(s)=a_{0}+a_{1} s+\cdots a_{n-1} s^{n-1}+s^{n} \\
& \text { with } \underline{a}_{i} \leq a_{i} \leq \bar{a}_{i} \text { for } 0 \leq i \leq n-1
\end{aligned}
$$

and

$$
\begin{aligned}
& b(s)=b_{0}+b_{1} s+\cdots b_{n-1} s^{n-1}+s^{n} \\
& \text { with } \underline{b}_{i} \leq b_{i} \leq \bar{b}_{i} \text { for } 0 \leq i \leq n-1
\end{aligned}
$$

respectively. By a slight abuse of notation, we shall use the notation $\mathcal{A}, \mathcal{B}$ to denote these polynomial families also.

Now, Theorem 2.3 establishes that all of the rational functions in $\mathcal{B} / \mathcal{A}$ are strictly positive real if and only if the functions

$$
\begin{aligned}
& \frac{k_{2}^{\mathcal{B}}(s)}{k_{1}^{\mathcal{A}}(s)}, \frac{k_{3}^{\mathcal{B}}(s)}{k_{1}^{\mathcal{A}}(s)}, \frac{k_{1}^{\mathcal{B}}(s)}{k_{2}^{\mathcal{A}}(s)}, \frac{k_{4}^{\mathcal{B}}(s)}{k_{2}^{\mathcal{A}}(s)}, \\
& \frac{k_{1}^{\mathcal{B}}(s)}{k_{3}^{\mathcal{A}}(s)}, \frac{k_{4}^{\mathcal{B}}(s)}{k_{3}^{\mathcal{A}}(s)}, \frac{k_{2}^{\mathcal{B}}(s)}{k_{4}^{\mathcal{A}}(s)}, \frac{k_{3}^{\mathcal{B}}(s)}{k_{4}^{\mathcal{A}}(s)},
\end{aligned}
$$

are strictly positive real. The result now follows from Theorem 2.1.

\section{Remarks:}

(i) The above result provides simple conditions that are necessary and sufficient for CQLF existence for a pair of LTI systems in companion form 
subject to interval uncertainty. In fact, it is only necessary to calculate the eigenvalues of eight matrix products, whereas testing for strict positive realness requires evaluating transfer functions at infinitely many values of $\omega$.

(ii) Note that in Corollary 3.1 the matrices $A \in \mathcal{A}$ and $B \in \mathcal{B}$ are related by $B=A-g h^{T}$ and not independent. This is the reason why in Corollary 3.1 we need only four fixed entries matrices whereas in Theorem 3.2 we need eight such matrices.

\section{Concluding remarks}

In this paper, we have considered the stability of switched linear systems subject to interval uncertainty. In particular, we have presented two results on common quadratic Lyapunov function existence for pairs of LTI systems with interval uncertainty in the entries of their system matrices. These results provide verifiable conditions for CQLF existence for interval matrix families in companion form. A numerical example has been presented to illustrate how the results of the paper can be used in practice.

\section{Acknowledgements}

This work was partially supported by Science Foundation Ireland Grant 03/RP1/I382 and Science Foundation Ireland Grant 04/IN1/I478. Science Foundation Ireland is not responsible for any use of data appearing in this publication. It was also partially supported by the Fund for the Promotion of Research at the Technion-Israel Institute of Technology.

\section{References}

Agrachev, A. A. and Liberzon, D. (2001). Lie-algebraic stability criteria for switched systems. SIAM Journal of Control and Optimization, 40(1), 253-269. 
Ando, T. (2001). Sets of matrices with a common Lyapunov solution. Archiv der Mathematik, 77, 76-84.

Bocher, M. (1964). Introduction to Higher Algebra. Dover, New York.

Buchberger, B. (1985). Recent Trends in Multi-dimensional Systems Theory, chapter "Grobner Bases: an algorithmic method in polynomial ideal theory". Reidel.

Chapellat, H., Dahleh, M., and Bhattacharyya, S. (1991). On robust nonlinear stability of interval control systems. IEEE Transactions on Automatic Control, 36(1), 59-67.

Cohen, N. and Lewkowicz, I. (2003). A pair of matrices sharing common Lyapunov solutions - a closer look. Linear Algebra and its Applications, 360, 83-104.

Gurvits, L., Shorten, R., and Mason, O. (2004). Preliminary results on the stability of switched positive linear systems. Submitted to Mathematical Theory of Networks and Systems.

King, C. and Nathanson, M. (2004). On the existence of a common quadratic Lyapunov function for a rank one difference. arXiv:math.OC/040346\%.

Liberzon, D. and Morse, A. S. (1999). Basic problems in stability and design of switched systems. IEEE Control Systems Magazine, 19(5), 59-70.

Meyer, K. (1966). On the existence of Lyapunov functions for the problem of Lur'e. J. SIAM Control, 3(3), 373-383.

Narendra, K. S. and Balakrishnan, J. (1994). A common Lyapunov function for stable LTI systems with commuting $\mathcal{A}$-matrices. IEEE Transactions on automatic control, 39(12), 2469-2471.

Narendra, K. S. and Goldwyn, R. M. (1964). A geometrical criterion for the stability of certain non-linear non-autonomous systems. IEEE Transactions on Circuit Theory, 11(3), 406-407.

Ooba, T. and Funahashi, Y. (1997). Two conditions concerning common quadratic Lyapunov functions for linear systems. IEEE Transactions on Automatic Control, 42(5), 719-721. 
Rojas, J. and Collada, J. (1994). Stability of interval matrices using the distance to the set of unstable matrices. In Proc. of the American Control Conference, pages 238-239, Maryland.

Sezer, M. and Siljak, D. (1994). On stability of interval matrices. IEEE Transactions on Automatic Control, 39(2), 368-371.

Shorten, R. N. and Narendra, K. S. (2003). On common quadratic Lyapunov functions for pairs of stable LTI systems whose system matrices are in companion form. IEEE Transactions on Automatic Control, 48(4), 618621.

Shorten, R. N., Narendra, K. S., and Mason, O. (2003). A result on common quadratic Lyapunov functions. IEEE Transactions on Automatic Control, 48(1), 110-113.

Shorten, R. N., Curran, P., and Wulff, K. (2004a). On time-domain multiplier criteria for single-input single-output systems. International Journal of Control, 77(11), 985-991.

Shorten, R. N., Mason, O., Cairbre, F. O., and Curran, P. (2004b). A unifying framework for the SISO Circle Criterion and other quadratic stability criteria. International Journal of Control, 77(1), 1-8.

Vilaplana, M., Mason, O., Leith, D., and Leithead, W. (2005). Switching and Learning in Feedback Systems, chapter "Control of yaw-rate and sideslip in 4-wheel steering cars with actuator constraints". Number LNCS 3355. Springer-Verlag, Lecture Notes in Computer Science.

Walach, E. and Zeheb, E. (1980). Sign test of multivariable real polynomials. IEEE Transactions on Circuits and Systems, 27(7), 619-625.

Zeheb, E. (1990). Necessary and sufficient conditions for robust stability of a continuous system-the continuous dependency case illustrated via multilinear dependency. IEEE Trans. on Circuits and Systems, 37(1), $47-53$. 